\title{
Steves' Review of Nuclear Medicine Technology: Preparation for Certification Examinations
}

\author{
N.E. Bolus and A.B. Brady \\ Reston, VA: Society of Nuclear Medicine Technology Section, 2011, 356 pages, $\$ 99$
}

Earlier editions of Review of Nuclear Medicine Technology: Preparation for Certification Examinations were a must-have for anyone taking the NMTCB or ARRT boards in nuclear medicine technology. On the retirement of Prof. Ann Steves, the NMT program director at the University of Alabama Birmingham and the original author, the task of authoring this important review book was taken on by her replacements at the University of Alabama Birmingham, Norman Bolus and Amy Brady. Both have done an excellent job in updating this fourth edition, and as a tribute to the work and dedication of Prof. Steves, they have included her name in the title.

In this edition, the authors have added some muchneeded content relating to PET, CT, informatics, and research techniques. This addition has increased the number of chapters from 16 to 19 in the main section. They have also added 3 new chapters in a new section titled "Advanced Certification and Post Primary Certification Primers," covering the nuclear cardiology, advanced PET certificate, and MRI primers. The appendices have gone from 3 to 6 , with the addition of a dual-isotope counting spilldown ratio data sheet, helpful Web links, and an electrocardiogram interpretation guide. Not to be outdone, they have also included 2 extra trial examinations, taking the number from 5 in edition 3 to 7 in this edition. These additions take the text from the third edition's 252 pages to 356 pages in this edition-an impressive job covering all the areas for which I, as an educator, was looking.

As my students say when they first receive this book, "This is all we needed; why did we need to buy the Cherry, the Christain, the Gopal...." A review book cannot cover all the required facts and herald the full understanding needed by students studying to be technologists in the field of nuclear medicine. However, it can be a wonderful tool in work toward the final step of the educational process, the board examination. This review book does exactly that. It is a tool for preparation just like the Kaplan or Princeton courses and review books for any of the standardized tests used for entrance into college or a specialty.

For students who have the proper foundation, the book is well written and easy to follow. It covers all necessary

COPYRIGHT @ 2012 by the Society of Nuclear Medicine, Inc.

Published online Jan. 25, 2012.

DOI: 10.2967/jnmt.111.100669 topics and gives easy access to all formulas, values, and regulatory amounts that students are required to memorize. One major plus is the trial examinations and their answers. Each answer has an explanation, along with a reference from a commonly used nuclear medicine textbook. My instructions to students whom I prepare for the board examination are the following 9 steps:

1. Review the NMTCB and ARRT requirements for each area being studied. Make sure you are familiar with all terms and studies referenced.

2. Read the Steves' text for each subject. As a quick review guide, create lists and tables with facts and figures of all the radiopharmaceuticals, studies, formulas, regulations, and anything else pertinent.

3. Make notes during steps 1 and 2 of any topic areas or information with which you are not familiar. Research those areas in your broader textbooks or ask the program director and clinical staff for assistance.

4. Once the text and subjects have been thoroughly covered and you have spent a few weeks repeatedly reviewing the topics, it is time to start taking the trial examinations.

5. Take the first examination, with the book closed and without cheating.

6. Review your answers, and see where there are gaps in your understanding.

7. Target your studying to the topics you struggled with in the first examination. Use the references supplied by the answers, and then review the Steves' text. Update your tables and lists accordingly. Review your lists and tables for the next few days.

8. Take the next examination, with the book closed and without cheating.

9. Repeat steps 5-7 for the rest of the trial examinations in the book.

The practice of nuclear medicine technology has changed dramatically since the publication of the third edition of this text in 2004. The board examinations now reflect many of those changes, and students can rely on the Steves' review book to address those changes. For example, contrast agents have been added to the patient care chapter, along with the latest guidelines for basic life support that are now required by all hospitals for health-care providers. In chapter 3, titled "Radiopharmaceuticals, Interventional 
Drugs and Introduction to Pharmacology," a section on pharmacology has been added, along with an invaluable table summarizing the uptake mechanism of all commonly used radiopharmaceuticals. The authors made a good call in expanding the former chapter 4, "Instrumentation," to 3 separate chapters: "Math and Radiation Physics Primer," "Instrumentation and Basic Counting Statistics," and "Instrumentation Quality Control." They have also added an entirely new chapter, chapter 7 , dedicated to positron CT and CT. Another addition to the text appears in chapter 8, which covers health informatics and introduces research methods. Chapter 10, which centers on specific organ systems and therapy, is similar in layout and title to the third edition. The only changes were minor, with the addition of new imaging studies and therapies in each area.
This textbook continues to be a must-have for anyone preparing to take the NMTCB or ARRT board examination, and I also recommend that those already certified take a look. It gives even the most seasoned technologist a basic overview of some new developments in the field. The authors have done an excellent job of updating this text, following the same format started by their colleague and predecessor, Prof. Steves.

Frances Keech

MA College of Pharmacy and Health Sciences 179 Longwood Ave., Griffin Building 414

Boston, MA 02115

E-mail: frances.keech@mcphs.edu

\section{Question 1}

\section{Answers to the Questions on Pages 66 and 67}

Answer $=$ B

A. Incorrect. Although traumatic fractures will initially be metabolically active while healing, the distribution is too random to be considered traumatic.

B. Correct. Malignant cells have increased glucose metabolism and will be detected because of uptake and intracellular trapping of ${ }^{18}$ F-FDG-6-phosphate. The pattern of distribution and the presence of focal bone lesions on the CT scan are most consistent with widespread osseous metastases.

C. Incorrect. Degenerative changes are an unlikely explanation as most of the ${ }^{18} \mathrm{~F}-\mathrm{FDG}$ bone distribution is clearly nonarticular. The CT scan does not demonstrate significant degenerative changes.

D. Incorrect. Although bone marrow stimulation, commonly seen after chemotherapy or administration of granulocyte colony-stimulating factor, affects the axial and proximal appendicular skeleton, its distribution is usually diffuse.

\section{Question 2}

Answer $=\mathbf{C}$

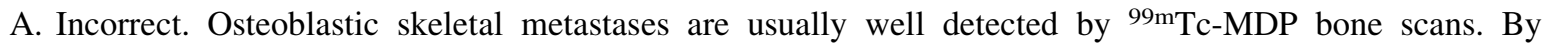
contrast, ${ }^{18} \mathrm{~F}-\mathrm{FDG}$ PET is reported as being less sensitive in detecting osteoblastic skeletal metastases than bone scintigraphy, such as in prostate carcinoma staging.

B. Incorrect. Widespread ${ }^{18}$ F-FDG PET bone activity with negative bone scan results is not typical of a benign process.

C. Correct. The best explanation in this patient for avid ${ }^{18} \mathrm{~F}-\mathrm{FDG}$ bone lesions poorly visualized on ${ }^{99 \mathrm{~m}} \mathrm{Tc}-$ MDP bone scans is that the metabolically active metastases have not yet generated a reactive osseous response detectable with ${ }^{99 \mathrm{~m}} \mathrm{Tc}-\mathrm{MDP}$ bone scintigraphy. The CT scan shows slightly hyperdense bone lesions in a medullary rather than cortical distribution. Early metastatic deposits first localize to the marrow.

D. Incorrect: Although non-small cell lung cancer is aggressive and often causes osteolytic metastases, this patient shows no CT evidence of lytic bone lesions. 